\section{In the news}

\section{SILENT COMMUNICATION}

Whether patients in a vegetative state are conscious and aware of their surroundings is a question that has long troubled families and doctors. Recent functional MRI (fMRI) studies involving a 23-year-old woman who sustained a severe traumatic brain injury have indicated that she might be aware of her surroundings and able to carry out mental tasks, prompting hopes that patients in vegetative states could one day communicate with those around them.

During an fMRI study, Adrian Owen et al. gave the patient spoken instructions to imagine walking around rooms at home or playing tennis. Owen described the neural reponses as "indistinguishable" from healthy volunteers, and claims that "her decision to work with us when asked represents a clear act of intent ... she was consciously aware of herself and her surroundings" (The Times, 8 September 2006). Writing in the same issue of Science, Lionel Naccache of the Cognitive Neuroimaging Unit in Orsay, France, agrees that "the fMRI findings indicate the existence of a rich mental life."

However, Owens himself points out that "all vegetative patients are different: they have damage done to different parts of their brains and their chances of recovery are different" (BBC News Online,

7 September 2006). Paul Matthews, at the University of Oxford, goes further: "When patients are in a vegetative state they can react to stimuli but not in a truly meaningful way ... Response to stimuli, even complex linguistic stimuli, does not provide evidence of a decision to respond" (The Times, 8 September 2006).

Regardless of the precise extent of awareness indicated by this study, as Naccache puts it: "this single case makes a strong argument for the development of fMRI and other neurophysiological tools ... to evaluate cognition in such patients", and might help to make decisions about patient care.
NEURODEGENERATIVE DISEASES

\section{Perils of ageing}

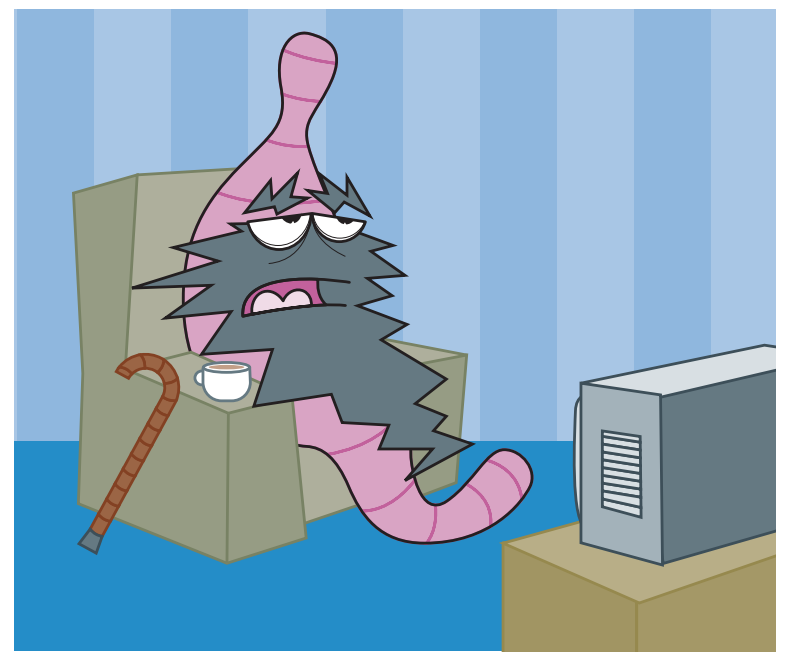

A molecular mechanism has now been proposed that explains why Alzheimer's disease (AD), which typically strikes in 50-80-year olds, is linked with ageing. Reporting in Science, researchers showed that the transcription factors HSF-1 and
DAF-16, which are regulated by a central ageing pathway, have opposing disaggregation and aggregation activities that function together to prevent $\mathrm{AD}$.

$\mathrm{AD}$, like other late-onset neurodegenerative diseases, is correlated with toxic aberrant protein aggregation - specifically, amyloid precursor protein breaks down into $A \beta_{1-42}$ peptides, which aggregate. But why aggregate-mediated toxicity is linked with age has remained unclear.

Cohen, Bieschke and colleagues investigated whether increasing the lifespan (or slowing the ageing) of Caenorhabditis elegans would delay the onset of aggregation. If so, then the late onset of $\mathrm{AD}$ could be due to a detoxifying activity that becomes compromised with ageing. If not, a stochastic time-related buildup of toxic aggregates to a threshold could explain the late onset of AD.

To distinguish between these possibilities, researchers disrupted the insulin signalling pathway, which is central in the regulation of ageing in worms, flies and mammals. In C. elegans, a sole insulin receptor, DAF-2, transduces a signal that

\title{
$\Rightarrow$ DEVELOPMENT
}

\section{Glutamate signals growth}

The involvement of glutamate in early brain development has been somewhat contentious: although a large body of in vitro evidence highlights signalling roles for glutamate during proliferation, migration, differentiation and survival, genetic disruption to glutamatergic activity has little or no effect on brain development. New work by Matsugami and colleagues goes some way to resolving this issue and provides compelling in vivo evidence that glutamatergic activity is vital for early developmental events. The absence of an effect following genetic disruption to glutamate receptors or glutamate release might reflect the compensatory action of other neurotransmitters during development. To overcome this potential confound, Matsugami and co-workers adopted the opposite approach: they overstimulated glutamate receptors in mice by knocking out the glutamate transporters GLAST and GLT1, which normally maintain low levels of extracellular glutamate. These knockout mice had multiple brain defects in the cortex, hippocampus and olfactory bulb after embryonic day (E) 15 and died by E17-18.

By E16, the number of cells in the ventricular zone - a layer of mitotic cells that eventually give rise to all cell types in the mature brain - was decreased in mutant brains compared with wild-type brains. The proportion

The migration pattern of newly generated neurons, darkly stained (BrdU incorporation experiments), was normal in the neocortex of wild type animals (top panel), but abnormal in the Glast $^{-1-} /$ Glt1 $^{-1-}$ E16 neocortex (bottom panel). Images courtesy of T. R. Matsugami, Tokyo Medical and Dental University, Japan.

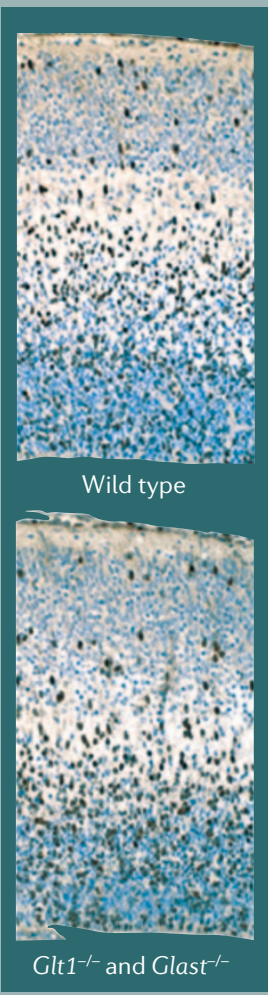


reduces the expression of genes that are regulated by the transcription factors DAF-16 and HSF-1, resulting in a shortened lifespan. By knocking down daf-2 in C. elegans that expressed human $A \beta_{1-42}$, the researchers showed that worms with longer lifespans had reduced toxic aggregation - late onset is therefore due to a compromised detoxifying activity rather than a stochastic accumulation. Double knockdown of $d a f-2$ with either $d a f-16$ or $h s f-1$, however, reversed this effect.

So, how do DAF-16 and HSF-1 inhibit the toxicity of protein aggregation? Examining the amounts of high molecular weight $A \beta_{1-42}$ aggregates and small $\mathrm{A} \beta_{1-42}$ aggregates, Cohen and colleagues made several interesting findings. First, HSF-1 regulates the disaggregation of $A \beta_{1-42}$ aggregates, but DAF-16 does not. By contrast, DAF-16 mediates the formation of high molecular weight $\mathrm{A} \beta_{1-42}$ aggregates, but these aggregates do not correlate with toxicity. Last, small $A \beta_{1-42}$ aggregates correlate with toxicity.

Together, these results are indicative of a mechanism that links ageing with late-onset $\mathrm{AD}$. As aggregates develop, HSF-1 activity mediates their disaggregation. DAF-16 activity supports an alternative pathway (which perhaps functions as a backup pathway) that mediates the formation of low toxicity, high molecular weight aggregates from high toxicity small aggregates. Because both detoxification pathways are mediated by the ageing-related insulin signalling pathway, both can become compromised with ageing, leading to aggregate build-up.

Interestingly, as the insulin signalling pathway is also associated with the formation of other toxic aggregates, such as those responsible for Huntington's disease, further research into this pathway could yield therapeutic targets for the general prevention of late-onset aggregation-linked neurodegenerative diseases.

Asher Mullard, Copy Editor

Nature Reviews Molecular Cell Biology

ORIGINAL RESEARCH PAPER Cohen, E. et al.

Opposing activities against age onset

proteotoxicity. Science 10 August 2006

(doi:10.1126/science.1124646) of proliferating cells in the ventricular zone (detected by BrdU labelling) was reduced in mutant brains, but the amount of cell death was unchanged, suggesting that extracellular glutamate concentration modulates neurogenesis at E16.

A laminar organization of the neocortex is usually seen by E16, but this pattern was severely disturbed in the brains of mutant mice, suggesting an impairment of cortical cell migration from the ventricular zone to other layers. BrdU labelling confirmed the presence of migration defects. Moreover, antibody staining revealed a disruption of radial glial fibres, which normally guide postmitotic neurons during migration. Neuronal tracing experiments with a fluorescent dye showed that these guidance defects resulted in severe disruptions to corticothalamic and thalamocortical pathways.

To confirm the involvement of excess glutamatergic signalling in these developmental defects, the authors administered antagonists of NMDA

\section{IN BRIEF}

\section{$\Rightarrow$ NEUROTRANSMITTERS}

Deletion of the GABA receptor $\alpha 1$ subunit increases tonic $\mathrm{GABA}_{\mathrm{A}}$ receptor current: a role for GABA uptake transporters.

Ortinski, P. I. et al. J. Neurosci. 26, 9323-9331 (2006)

Mice lacking the gene for the $\alpha 1$ GABA $(\gamma$-aminobutyric acid) receptor subunit type $A\left(G A B A_{A}\right)$, a crucial component of GABA -mediated synaptic neurotransmission, exhibit remarkable functional compensation: despite missing more than half the normal number of $\mathrm{GABA}_{A}$ receptors they show no overt phenotype. Ortinski et al. now show the mechanism of compensation to be an increase in 'tonic' $\mathrm{GABA}_{\mathrm{A}}$ receptormediated current through high-affinity non- $\alpha 1$-containing receptors. The abundance of these receptors was unchanged, but the level of extrasynaptic GABA was increased owing to a decrease in GABA transporter activity.

\section{$\Rightarrow$ NEUROGENESIS}

Neocortical neurogenesis in humans is restricted to development.

Bhardwaj, R. D. et al. Proc. Natl Acad. Sci. USA 103, 12564-12568 (2006)

Debate over the existence of adult-born neurons in the neocortex is compounded by difficulties in assessing neurogenesis in humans. Now, evidence has emerged showing that, in the human cortex, no new neurons are added after birth. The authors employed a technique that takes advantage of changing levels of atmospheric ${ }^{14} \mathrm{C}$ during the twentieth century. From 1955 to $1963,{ }^{14} \mathrm{C}$ levels increased owing to nuclear bomb testing, then rapidly declined. These changes are reflected in the ${ }^{14} \mathrm{C}$ content of DNA in cells becoming postmitotic during these periods. In post-mortem brain tissue, all neocortical neurons examined were demonstrated to have been present at birth, although the authors note that neurogenesis contributing to less than $1 \%$ of the total neocortical population would not have been detected by this method. These findings, together with evidence from BrdU studies, provide further evidence to suggest that neurons are not normally generated in the adult neocortex.

signalling through these receptors does indeed modulate early brain developmental processes, but also that other mechanisms are important for these processes.

It will be interesting to determine which other neurotransmitter systems have a role in early brain development and to unravel the separate contributions of neuronal activity and genetic programming. The use of excess stimulation, in addition to genetic ablation, of neurotransmitter pathways might be one way to answer these questions.

\section{Daniel McGowan}

ORIGINAL RESEARCH PAPER Matsugami, T. R.

et al. Indispensability of the glutamate transporters GLAST and GLT1 to brain development. Proc. Natl Acad. Sci. USA 103, 12161-12166 (2006)

\section{NEURODEGENERATIVE DISEASES}

Ubiquitin hydrolase Uch-L1 rescues $\beta$-amyloidinduced decreases in synaptic function and contextual memory.

Gong, B. et al. Cell 126, 775-788 (2006)

The ubiquitin-proteasome pathway is known to have a role in the pathogensis of Alzheimer's disease (AD). It now seems that inhibiting the activity of a component of this pathway, ubiquitin C-terminal hydrolase L1 (Uch-L1), is associated with impairments in long-term potentiation in slice cultures treated with oligomeric A $\beta$. Increasing Uch-L1 activity through treatment with exogenous Uch-L1 reversed this effect in the hippocampal slice cultures and in slices from App/PS1 mice. Moreover, in App/PS1 mice, exogenous delivery of Uch-L1 led to an improvement in contextual memory. Further work showed that the effect of Uch-L1 on synaptic function depended on the protein kinase A (PKA)cyclic AMP response element binding (CREB) protein pathway. These findings highlight a potential therapeutic target for the memory difficulties in AD. 